\title{
Keramik basierter all-solid-state pH-Sensor in Multilayertechnologie
}

\author{
Claudia Feller, Horst Griessmann, Kathrin Reinhardt, Alexander Michaelis
}

Fraunhofer-Institut für Keramische Technologien und Systeme, Dresden, Winterbergstraße 28

\begin{abstract}
Auf Basis eines keramischen HTCC-Tapes wurde ein aus einer all-solid-state pH- und Referenzelektrode bestehender potentiometrischer pH-Sensor im Mehrlagenaufbau entwickelt. Die elektrochemische Charakterisierung der separaten Halbzellen und des Sensors erfolgte im pH-Bereich von 1,68 pH bis 9,18 pH bei Temperaturen von 25 bis $65^{\circ} \mathrm{C}$. Untersuchungen zur Potentialstabilität der Elektroden und des Sensors wurden in Abhängigkeit von Zeit und Aufbewahrungsmedium durchgeführt. Dabei wurde zwischen einer Lagerung in destilliertem Wasser, gesättigter KCl-Lösung und einer Trockenlagerung unterschieden. Außerdem wurde der Einfluss von Dampfsterilisationszyklen auf die Eigenschaften von pH- und Referenzelektrode untersucht. In einem Untersuchungszeitraum von 4 Wochen besitzt der Sensor eine sehr gute Messperformance bezüglich Empfindlichkeit, Ansprechzeit und Potentialstabilität.
\end{abstract}

\section{Einleitung}

Das Potential, das die Multilagentechnologie material- und technologieseitig bietet, ist für die Entwicklung elektrochemischer Sensoren insbesondere für die Größe pH-Wert vorteilhaft nutzbar. Vorteile keramischer Multilagen basierter Sensoren sind deren durch den 3-D-Aufbau bedingte hohe Integrationsrate von Sensorkomponenten, mechanische Robustheit, hohe chemische Beständigkeit sowie Dichtheit und die damit verbundene geringe Störanfälligkeit gegenüber Quereinflüssen. Unter Anwendung der Folien- und Multilayertechnolgie ist ein Keramik basierter all-solid-state $\mathrm{pH}$-Sensor realisiert worden, der aufgrund seiner Messperformance zur pH-Wertkontrolle in zahlreichen analytischen und industriellen Verfahren einsetzbar ist.

\section{Aufbau des Sensors}

Alle inneren Funktionselemente des Sensors werden durch Siebdruck bzw. Strukturierung der einzelnen Grünfolien aufgebracht (Bild 1). Anschließend werden die Grünfolien durch Laminieren und Sintern in ein Mehrlagensystem überführt. Auf das gebrannte Substrat werden nachfolgend die sensoraktiven Schichten

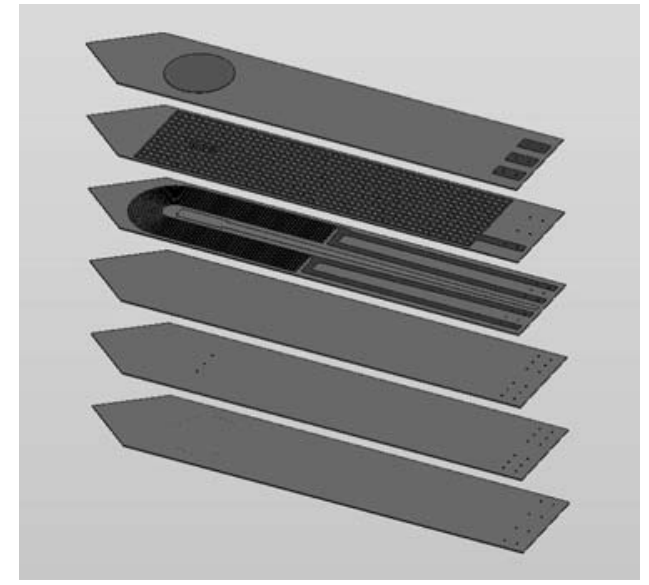

Bild 1 3D-Darstellung des $\mathrm{pH}$-Sensors, Aufbau Lagen Halbzelle pH-Elektrode

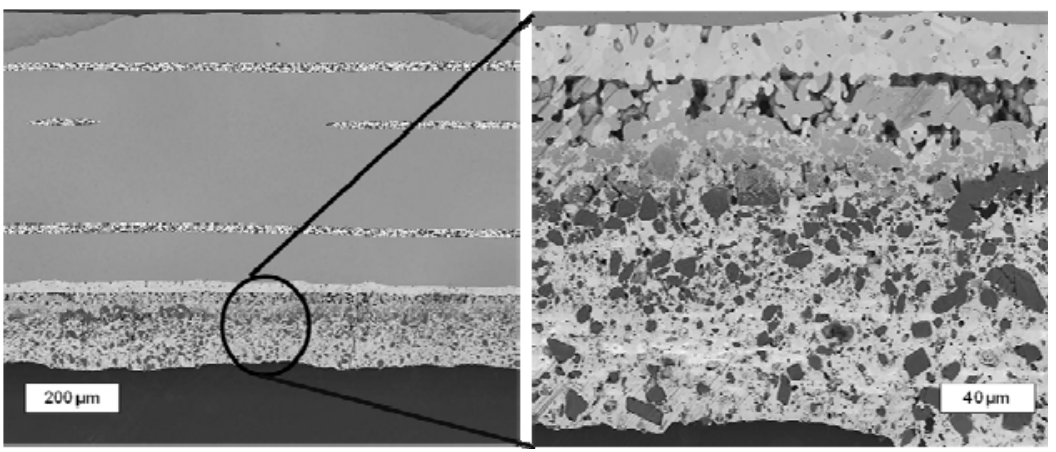

Bild 2 FESEM-Aufnahme der Halbzelle Referenzelektrode, Chloridreservoir 
siebgedruckt. Als pH-sensitive Schicht wurde ein im thermischen Ausdehnungskoeffizienten zur HTCCKeramik kompatibles Glas ausgewählt. Die Referenzelektrode basiert auf dem System Ag/AgCl/NaCl, wobei das Chloridreservoir aus einem Salz-Glas-Gemenge besteht (Bild 2). Der keramische Mehrlagenaufbau gestattet es, alle Metallisierungsschichten in den inneren Keramiklagen zu vergraben und dadurch jeglichen Kontakt mit der Messlösung zu unterbinden. In Folge dessen werden Mischpotentiale vollständig ausgeschlossen. Desweiteren wurde im Inneren der Keramik unmittelbar über der pH-sensitiven Schicht ein Thermoelement Pt100 für die Temperaturmessung integriert. Der Mehrlagenaufbau erfolgte mit dem gewählten Layout im Fünffachnutzen.

\section{Ergebnisse und Diskussion}

\subsection{Halbzelle pH-Elektrode}

Die pH-Elektrode weist Nernst-Verhalten auf. Bild 3 zeigt das Ansprechverhalten in Wiederholungsmessungen. Die Messergebnisse werden in drei Zyklen sehr gut reproduziert. In Bild 4 ist die Potentialstabilität anhand der U-pH-Kennlinien dargestellt. Die wöchentlich gemessenen Kennlinien weisen eine sehr geringe Streuung auf. Im Untersuchungszeitraum von 12 Wochen wird mit dieser Elektrode der pH-Wert ohne Elektrodenkalibrierung mit einer maximalen Abweichung von 0,5 pH gemessen.

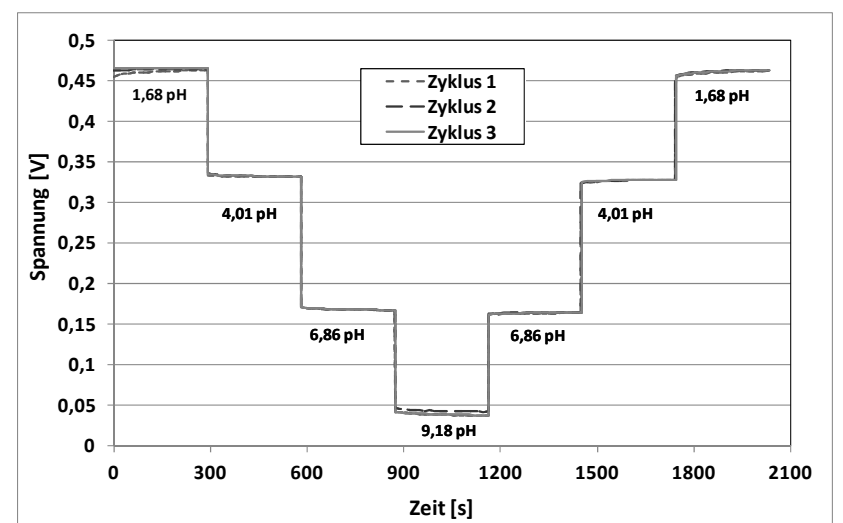

Bild 3 Ansprechverhalten einer pH-Elektrode bei $25^{\circ} \mathrm{C}$ in Wiederholungsmessungen mit drei Zyklen

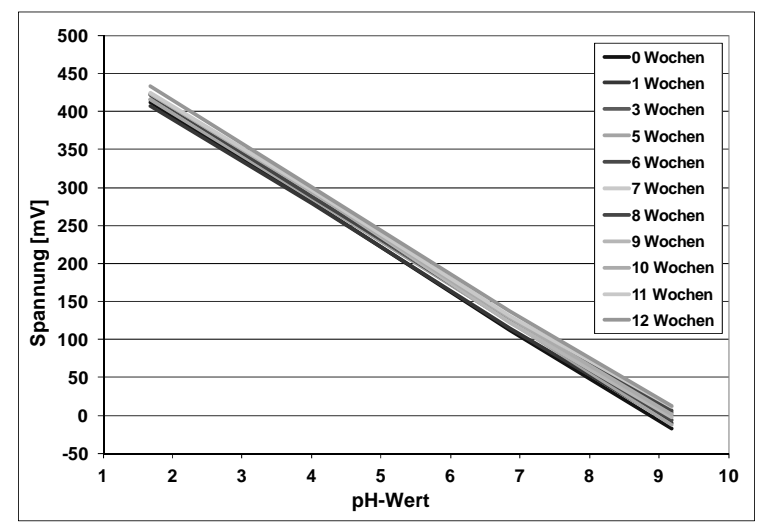

Bild 4 Kennlinienverlauf einer pH-Elektrode bei $25^{\circ} \mathrm{C}$ innerhalb von 12 Wochen

Im Hinblick auf eine langzeitstabile Referenzelektrode, die die Lebensdauer des pH-Sensors maßgeblich beeinflusst, wurden die Elektroden in destilliertem Wasser, gesättigter KCl-Lösung oder trocken gelagert. In Bild 5 sind die über den Zeitraum von 12 Wochen gemittelten Werte der pH-Empfindlichkeiten der Elektroden der einzelnen Serien dargestellt. Während die in Wasser und trocken gelagerten Elektroden Nernst-Empfindlichkeiten von über 95 \% aufweisen, besitzen die in KCl-Lösung aufbewahrten Elektroden niedrigere Empfindlichkeiten von etwa 90 \%. Die trocken gelagerten Elektroden haben ebenfalls die geringsten maximalen Abweichungen des gemessenen $\mathrm{pH}-$ Wertes (Bild 6).
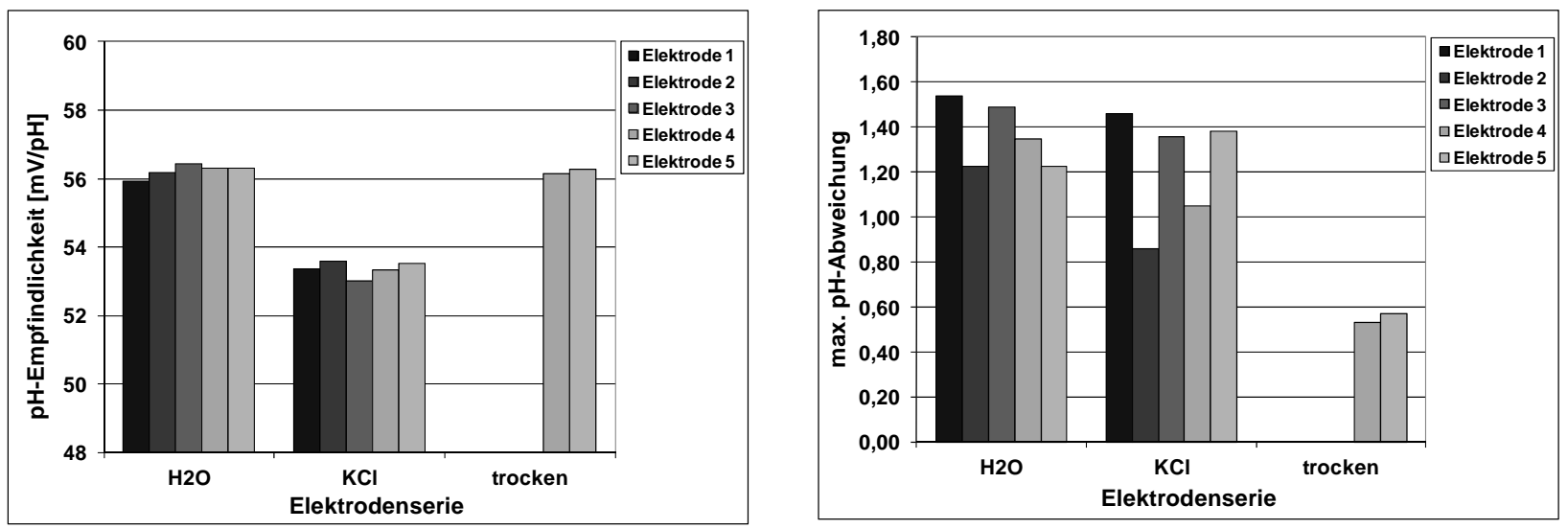

Bild 5 Mittlere pH-Empfindlichkeit bei $25^{\circ} \mathrm{C}$ in 12 Wochen Bild 6 Maximale pH-Abweichung bei $25^{\circ} \mathrm{C}$ in 12 Wochen 


\subsection{Halbzelle Referenzelektrode}

Ziel der Entwicklungsarbeiten ist es, eine langzeitstabile Referenzelektrode auf Basis eines mittels Siebdruck erzeugten, stabilen Chloridreservoirs zu entwickeln. Es wurden vier verschiedene Konfigurationen (A, B, C, D) untersucht, die sich in der Salzkonzentration des Chlorid-Glas-Reservoirs sowie in der Polymerabdeckung des Referenzmaterials unterscheiden. Die Elektroden wurden in 0,1 M KCl-Lösung ausgelagert und ihre Elektrodenpotentiale gegenüber einer konventionellen Ag/AgCl/KCl-Elektrode gemessen (Bild 7).

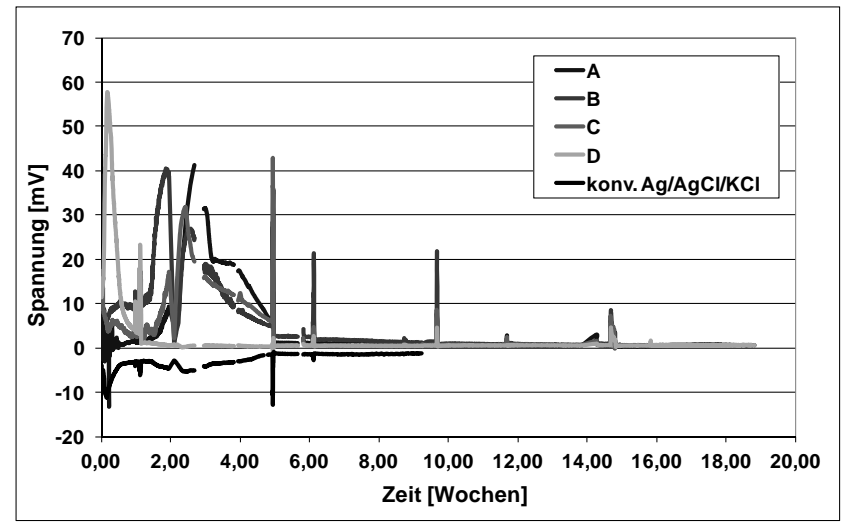

Bild 7 Potentialmessung in 0,1 M KCl-Lösung gegen eine konventionelle $\mathrm{Ag} / \mathrm{AgCl} / \mathrm{KCl}$-Elektrode bei $25^{\circ} \mathrm{C}$

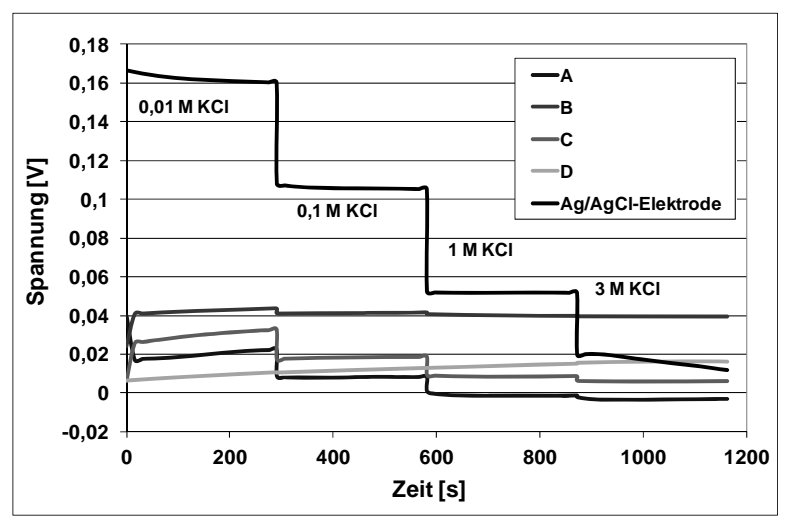

Bild 8 Chloridionenempfindlichkeit bei $25^{\circ} \mathrm{C}$

Zur besseren Bewertung der Messergebnisse wurde eine konventionelle Referenzelektrode über die ersten 9 Wochen mitgeführt. Die Potentiale der Referenzelektroden sollten, ein funktionsfähiges Chloridreservoir vorausgesetzt, nur wenige mV betragen. Die Referenzelektroden weisen zu Beginn der Auslagerung eine unterschiedlich starke Formierungsphase auf. Während die Referenzelektrode D bereits nach einer Woche ein stabiles Potential aufweist, wird für alle anderen Referenzelektroden erst ab der 5 . Woche nach einer thermischen Behandlung bei $65^{\circ} \mathrm{C}$ ein sehr stabiles Potential von wenigen $\mathrm{mV}$ gemessen. Die Auslagerungsversuche der Referenzelektroden sind bis zur 19. Woche fortgesetzt worden. In dieser Zeit wurde auch der Einfluss einer thermischen Belastung bei $65^{\circ} \mathrm{C}$ auf die Referenzelektroden getestet. Dies wird durch die „Spannungsspitzen“ bei 6, 10 und 15 Wochen angezeigt. Es ist deutlich zu erkennen, dass alle Referenzelektroden entsprechend der NERNST-Gleichung ihr Potential in Abhängigkeit von der Temperatur ändern. Nach Beendigung der thermischen Belastung besitzen alle Referenzelektroden wieder ein stabiles Potential bei $25{ }^{\circ} \mathrm{C}$. Die Auswertung zur Potentialstabilität der Referenzelektrode B ergab, dass diese Elektrode innerhalb der 7. bis 19. Auslagerungswoche eine Potentialdrift von weniger als $0,01 \mathrm{mV} / \mathrm{d}$ aufweist.

Zum Nachweis eines funktionsfähigen Chloridreservoirs ist die Chloridempfindlichkeit der Referenzelektroden bestimmt worden (Bild 8). Unter der Voraussetzung, dass $\mathrm{NaCl}$ im Reservoir gesättigt vorliegt und damit die Chloridionenaktivität konstant ist, ist auch das Elektrodenpotential bei $25^{\circ} \mathrm{C}$ konstant und die Elektrode als Bezugselektrode einsetzbar. Die Messungen erfolgten in unterschiedlich konzentrierten KClLösungen. Zur besseren Bewertung und zum Vergleich der Messergebnisse wurde eine Messung mit einer Ag/AgCl-Elektrode durchgeführt. Diese besitzt kein gesättigtes Chloridreservoir und demnach ist das Elektrodenpotential von der Chloridionenkonzentration abhängig. Das Ansprechverhalten der Ag/AgClElektrode ist vergleichend zum Ansprechverhalten der multilayerbasierten Referenzelektroden abgebildet. Die Elektroden B und D zeigen kaum eine Chloridionenempfindlichkeit, dagegen ändern sich die Potentiale der Elektroden A und C bei Änderung der Chloridkonzentration stärker. Daraus kann geschlussfolgert werden, dass die Konfigurationen der Elektroden B und D zu einem äußerst funktionsfähigen Chloridreservoir führen.

Im Hinblick auf zukünftige Anwendungen wurden die Halbzellen pH- bzw. Referenzelektrode bis zu 20-mal dampfsterilisiert (Bild 9). Die Dampfsterilisation erfolgte 10 min bei $130^{\circ} \mathrm{C}$ und 1,7 bar Wasserdampf. Nach jeder Sterilisation ist die Funktionsfähigkeit der Elektroden geprüft worden. Bis zur 20. Dampfsterilisation weist die pH-Elektrode Empfindlichkeiten von mehr als 90 \% auf. Die Funktionsfähigkeit der sterilisierten Referenzelektrode wurde jeweils durch eine Messung gegen eine konventionelle $\mathrm{pH}$-Glaselektrode geprüft. Die gemessenen pH-Empfindlichkeiten waren deutlich größer als $95 \%$. Bild 10 stellt den Verlauf der gemessenen Offset-Spannungen als Maß der Potentialstabilität nach den Sterilisationszyklen dar. Bei der pH- 
Elektrode treten relativ große Änderungen auf. Daraus folgt, dass die pH-Elektrode nach jeder Dampfsterilisation vor unmittelbarem Einsatz kalibriert werden muss. Dagegen sind die gegenüber der sterilisierten Referenzelektrode gemessenen Offset-Spannungen der konventionellen pH-Glaselektrode sehr stabil.

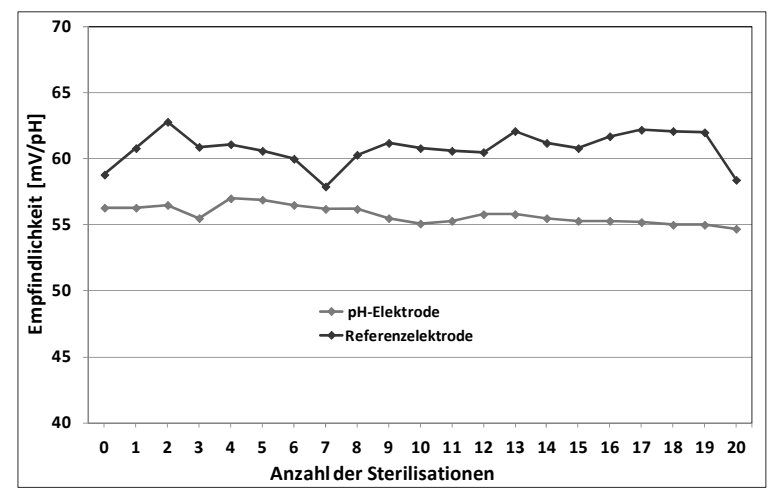

Bild 9 Empfindlichkeiten bei $25^{\circ} \mathrm{C}$ der dampfsterilisierten Elektroden

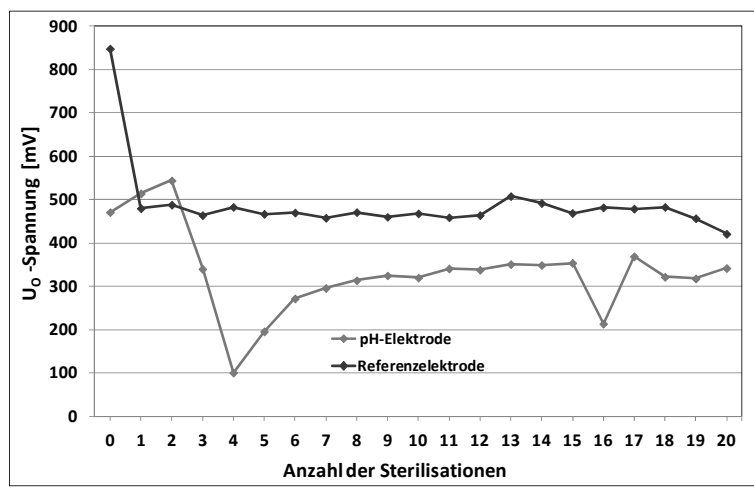

Bild 10 Offset-Spannungen bei $25^{\circ} \mathrm{C}$ der dampfsterilisierten Elektroden

\section{$3.3 \mathrm{pH}-$ Sensor}

Der pH-Sensor besitzt ein sehr gutes Nernst-Verhalten. Bild 11 zeigt das Ansprechverhalten des Sensors in Wiederholungsmessungen. Innerhalb der drei Zyklen werden die Messergebnisse sehr gut reproduziert. In Bild 12 ist die Potentialstabilität anhand der wöchentlich gemessenen Sensorkennlinien dargestellt. Zwischen den Messungen wurde der Sensor in gesättigter KCl-Lösung aufbewahrt. Die Kennlinien besitzen nach der anfänglichen Formierung der Referenzelektrode ab der 1. Woche eine geringe Streuung. Von der 1. bis zur 4 . Woche beträgt die maximale Abweichung des gemessenen $\mathrm{pH}$-Wertes $0,4 \mathrm{pH}$.

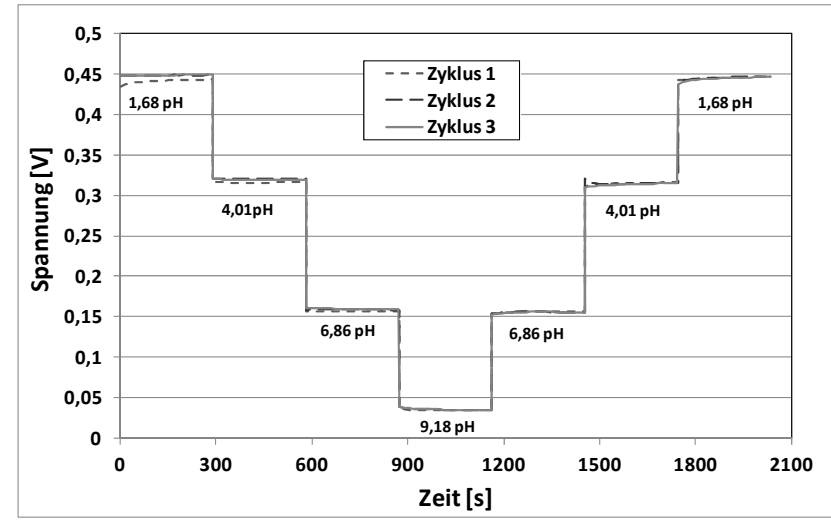

Bild 11 Ansprechverhalten eines pH-Sensors bei $25^{\circ} \mathrm{C}$ in Wiederholungsmessungen mit drei Zyklen

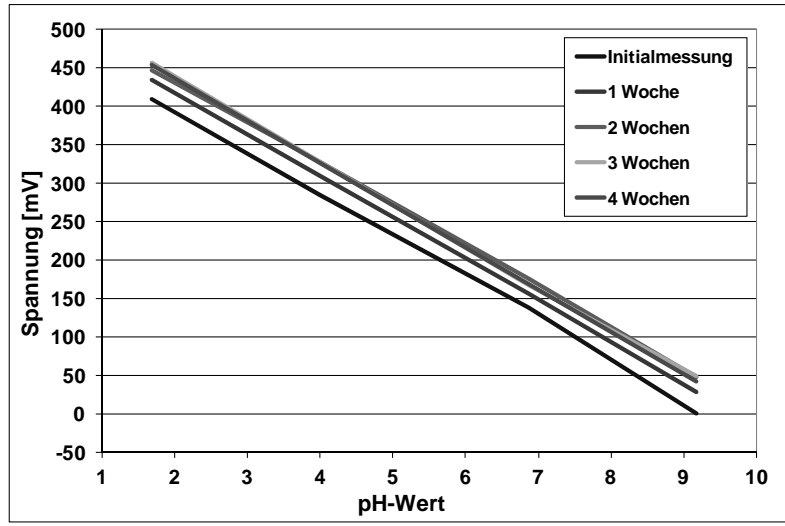

Bild 12 Kennlinienverlauf eines pH-Sensors bei $25^{\circ} \mathrm{C}$ innerhalb von 4 Wochen

\section{Zusammenfassung}

Basierend auf der HTCC-Multilayertechnologie ist ein $\mathrm{pH}$-Sensor entwickelt worden, der $\mathrm{pH}$ - und Referenzelektrode in einem Mehrlagensystem vereint. Der Multilayer basierte pH-Sensor weist eine exzellente Messperformance bezüglich Ansprechzeit, Empfindlichkeit und Potentialstabilität auf. Sowohl pH-Elektrode als auch Referenzelektrode können bei Erhaltung ihrer Eigenschaften bis zu 20 Sterilisationszyklen unterworfen werden. Die Lebensdauer der beiden Halbzellen pH-Elektrode und Referenzelektrode betragen entsprechend dem aktuellen Untersuchungsstand mehr als ein Jahr.

\section{Danksagung}

